\title{
Evaluation of Silages of Potato, Sweet Potato and Turnip with Rice Straw or Wheat Straw with or without Urea and Studies the Effect of Rations Containing Its Silages on Digestion Coefficients and Rumen Fermentation in Sheep
}

\author{
Zaki, M. A. ${ }^{2}$; A. A. Osman' ${ }^{1}$; E. S. Soliman' and A. H. Ghoniem ${ }^{2}$ \\ ${ }^{1}$ Department of Animal Production, Faculty of Agriculture, Suez Canal University \\ ${ }^{2}$ Animal Production Research Institute, Agriculture Research Center, Egypt
}

Received: $21 / 5 / 2020$

\begin{abstract}
The objectives of this study were evaluation the silages containing of non-commercial potato tubers, sweet potato roots, and turnip roots. Silages were making manually in jars in the $1^{\text {st }}$ experiment with mixing cutting tubers or roots with rice straw or wheat straw with urea additives at levels $0,0.5 \%$ and $1 \%$ for determining chemical composition and fermentation characteristics of silages. In the $2^{\text {nd }}$ experiment, silages were making manually in bags with mixing cutting tubers or roots with rice straw $+2 \%$ molasses with 0 or $0.5 \%$ urea for evaluating seven rations by rams as follows: Ration A $100 \%$ of CP requirements according to NRC (1985) from concentrate feed mixture (CFM) + rice straw ad lib. Rations B, C and D were $60 \%$ of CP requirements from CFM + silages of potato, sweet potato and turnip, respectively. Rations $\mathrm{E}, \mathrm{F}$ and $\mathrm{G}$ were $60 \%$ of $\mathrm{CP}$ requirements from $\mathrm{CFM}+$ silages of potato, sweet potato and turnip containing $0.5 \%$ urea, respectively. Silages were fed ad lib. Digestion coefficients and rumen fermentation were conducted to evaluate rations A, B, C, D, E, F and G using 21 local rams (3 in each) averaged weight $49 \mathrm{~kg}$. Results explained that DM\% of potato tubers, sweet potato and turnip roots were 18.18, 20.08 and $7.50 \%$, respectively and $\mathrm{CP} \%$ was $13.31,10.46$ and $13.46 \%$, respectively. DM of potato silage ranged from 34.31 to $35.68 \%$, sweet potato silage ranged from 34.39 to $35.73 \%$ and turnip silage ranged from 31.57 to $37.71 \%$. CP in silage with urea was higher than silage without urea. Silage fermentation characteristics explained that $\mathrm{pH}$ _ranged from 3.80 to 4.20 , Ammonia-N\% of total $\mathrm{N}$ ranged from 9.61 to $16.22 \%$, Acetic acid ranged from 2.36 to $3.52 \mathrm{~g} / 100 \mathrm{~g} \mathrm{DM}$, Butyric acid ranged from 0.28 to $1.34 \mathrm{~g} / 100 \mathrm{~g}$ DM and lactic acid ranged from 6.31 to $9.65 \mathrm{~g} / 100 \mathrm{~g}$ DM. Ammonia-N as g/100g DM was increased with increasing urea levels. The differences of DM intake as \% of LBW among all rations containing silages were not significant. Digestion coefficients of $\mathrm{DM}, \mathrm{OM}$ of ration A was significantly $(\mathrm{P}<0.05)$ higher than all rations and the differences among other rations containing silages were not significant. Digestion coefficients of DM of rations containing silages ranged from 52.88 to $56.94 \%$, OM ranged from 54.69 to $59.03 \%$ and $\mathrm{CP}$ ranged from 58.61 to $64.8 \%$. TDN of control was 60.49 and other rations containing silages ranged from 51.61 to $55.83 \%$. DCP of control was 8.08 and other rations ranged from 7.79 to $9.58 \%$. Digestion coefficients and nutritive values were not affected with urea additives. Ruminal parameters indicated that the differences of ruminal $\mathrm{pH}$ among all rations were not significant at $4 \mathrm{~h}$ post feeding. The differences of $\mathrm{NH}_{3}-\mathrm{N}$ and Total VFA's among rations $\mathrm{B}, \mathrm{C}$ and $\mathrm{D}$ were not significant and the differences among rations $\mathrm{E}, \mathrm{F}$ and $\mathrm{G}$ were not significant at 2 and $4 \mathrm{~h}$ post feeding. The $\mathrm{NH}_{3}-\mathrm{N}$ and VFA's of rations containing silages with urea was significantly $(\mathrm{P}<0.05)$ higher than control and rations containing silages without urea. The differences of Microbial protein among all rations were not significant except ration B was lower than other rations.
\end{abstract}

Keywords: Potato, sweet potato, turnip, silage, urea, rams, digestion coefficients, ruminal parameters

\section{INTRODUCTION}

The shortage in animal local feed sources and high price of traditionally feeding especially concentrates are limiting animal production in Egypt. Therefore, the untraditionally feed is necessary for animal feeding. The crop residues such as corn stover, wheat straw and rice straw are utilizing in animal feeding with or without treatments. On the other side, by-products of roots and tubers could be utilizing in animal nutrition. The main problem in these products is produce in short time during harvesting of the crop. Moreover, these by-products had a high content of moisture. Leonel et al. (2017) found that DM of Potato tubers ranged from 11.89 to $21.83 \%$, Samy et al. (2014) found that the DM of different cultivars of sweet potato roots ranged from 17.0 to $26.5 \%$ and Penno et al. (1996) mentioned that DM of turnip roots ranged from 8.6 to $8.7 \%$. Therefore, these tubers and roots could be ensiled with dry crop residues such as rice straw and wheat straw for produce optimum DM in silages. Sadri et al. (2018) found that DM of silage contained potato and

\footnotetext{
*Corresponding author e-mail: mohamed.zaky@arc.sci.eg
}

wheat straw was $32.2 \%$, Mutavhatsindi et al. (2018) found that DM of silage contained Potato hash and wheat bran was $35.2 \%$, Babaeinasab et al. (2015) found that DM of silage contained Potato+wheat straw was $32.2 \%$ and Hart and Horn (1987) mentioned that DM of silage containing turnip and wheat straw was $33.2 \%$. Recently studies explained that silage fermentation characteristics of mixing tubers with crop residues lie in the good quality silage (Sadri et al., 2018; Rui-rui et al., 2018; Mutavhatsindi et al., 2018; Babaeinasab et al., 2015). HaiYan et al. (1998) noticed that no significant differences in ammonia-N concentration among different silages of turnip containing 6,12 and $18 \%$ rice straw. Adding molasses improved the ensiling fermentation of potato-wheat straw silage (Babaeinasab et al., 2015). Hart and Horn (1987) found that $\mathrm{pH}$ and $\mathrm{NH}_{3}$-Nwere increased and lactic acid was decreased with increasing levels of wheat straw in turnip ensiled with straw while the acetic and butyric acids not affected. Ruiz et al. (1981) found that acetic, butyric and lactic acids were fluctuated with different levels of 
urea in sweet potato silage treated with urea levels 0 , $0.4,0.8$ and $1.2 \%$. The silages containing tubers and roots were good palatability by ruminants (Sadri et al., 2018; Nkosi et al., 2010 with sheep and Aibibula, et al., 2007; Nelson et al., 2000 with cattle). A little information was found on digestion coefficient and rumen fermentation of rations containing silages of potato tubers or sweet potato and turnip roots. Sadri et al. (2018) found that digestion coefficients of DM and CP were 67.7 and $67.0 \%$ of ration containing $30 \%$ potato-wheat straw silage $+\mathrm{CFM}+$ alfalfa by sheep. Nkosi et al. (2010) found that digestion coefficients of DM and CP were 49.30 and $40.40 \%$ of silage containing $80 \%$ potato hash $+20 \%$ hay by sheep. Hart and Horn (1987) found that OM digestibility was $63.0 \%$, ruminal $\mathrm{pH}$ was 6.72 and ruminal total VFA was $82.6 \mathrm{mmol} / \mathrm{L}$ of sheep fed silage containing $72.3 \%$ turnip and $27.7 \%$ wheat straw.

However, there is limited information on ensiling these tubers and roots with or without additives. So, the aim of this study was evaluation the silages containing non-commercial potato tubers, sweet potato roots, and turnip roots with rice straw and wheat straw with or without urea additives and effect of rations containing its silages on digestion coefficients and rumen fermentation in sheep.

\section{MATERIALS AND METHODS}

This study was carried out at Animal Production Research Institute, Agricultural Research Center, Egypt. Two experiments were conducted:

\section{$1^{\text {st }}$ experiment for making silage in jars:}

This experiment was carried out at Animal Nutrition Unite of Ismailia Research Station (Ismailia governorate) (Animal Production Research Institute). Potato tubers, sweet potato roots and turnip roots were obtained from Ismailia market then cutting by using knives and mixed with chopped rice straw or wheat straw, then mixed with urea at levels $0,0.5$ and $1 \%$ on fresh basis in 18 treatments ( 9 with rice straw and 9 with wheat straw). The silage contents were calculated to give silage containing 35\% DM. Every mixture put in jar capacity $1 \mathrm{~kg}$ (three jars in each treatment) with good pressing, and then closes every jar tightly to provide a non aerobic environment. The jars were opened after 45 days for measuring chemical composition and silage fermentation characteristics.

\section{$2^{\text {nd }}$ experiment for making silage in bags:}

This experiment was carried out at Animal Nutrition Research Department (Animal Production Research Institute). Potato tubers, sweet potato roots and turnip roots were obtained from Aloboor market then cutting by using knives and mixed with chopped rice straw in six mixtures as follows:

$1-75 \%$ Potato and $25 \%$ rice straw $+2 \%$ molasses. $2-$ $75 \%$ sweet potato and $25 \%$ rice straw $+2 \%$ molasses. $3-$ $75 \%$ turnip and $25 \%$ rice straw $+2 \%$ molasses. $4-75 \%$ Potato and $25 \%$ rice straw $+2 \%$ molasses $+0.5 \%$ urea. $5-75 \%$ sweet potato and $25 \%$ rice straw $+2 \%$ molasses $+0.5 \%$ urea. $6-75 \%$ turnip and $25 \%$ rice straw $+2 \%$ molasses $+0.5 \%$ urea, then every mixture was putted into a plastic bag capacity $250 \mathrm{~kg}$ with a good pressing and still 45 days before opening. These silages were evaluated.

Seven experimental rations were evaluated by using rams as follows:

Ration A: $100 \%$ of CP requirements according to NRC (1985) from Concentrate Feed Mixture (CFM) + Rice straw ad lib.

Ration B: $60 \%$ of $\mathrm{CP}$ requirements according to NRC (1985) from CFM + silage of potato (1)

Ration C: $60 \%$ of $\mathrm{CP}$ requirements according to NRC (1985) from CFM + silage of sweet potato (2)

Ration D: $60 \%$ of $\mathrm{CP}$ requirements according to NRC (1985) from CFM + silage of turnip (3)

Ration E: $60 \%$ of $\mathrm{CP}$ requirements according to NRC (1985) from CFM + silage of potato contained $0.5 \%$ urea (4)

Ration F: $60 \%$ of $\mathrm{CP}$ requirements according to NRC (1985) from CFM + silage of sweet potato contained $0.5 \%$ urea (5)

Ration G: $60 \%$ of $\mathrm{CP}$ requirements according to NRC (1985) from CFM + silage of turnip contained $0.5 \%$ urea (6)

Digestibility trials were conducted to evaluate the rations $\mathrm{A}, \mathrm{B}, \mathrm{C}, \mathrm{D}, \mathrm{E}, \mathrm{F}$ and $\mathrm{G}$ using 21 Local rams (3 rams in each) averaged weight $49 \mathrm{~kg}$. Rams were individually housed in metabolic cages. Preliminary period was 21 days and collection period were 5 days, followed 3 days for rumen fermentation studies. Concentrate feed mixture (CFM) was daily offered to the animals in two equal portions at 8 am and $4 \mathrm{pm}$. The silages were weighed and offered $a d$ lib. Residual were collected and weighed daily. Drinking water was available all time.

Composite samples of CFM, rice straw, wheat straw, potato, sweet potato, turnip and silages of experimental jars and bags were dried in oven at $60^{\circ} \mathrm{C}$ for $24 \mathrm{~h}$. Samples of daily feces were collected and dried in oven at $60^{\circ} \mathrm{C}$ for $24 \mathrm{~h}$. Composite samples of feeds and feces were milling to pass through $1 \mathrm{~mm}$ screen and stored for chemical analysis. Chemical composition of representative samples was determined according to AOAC (1995) procedures.

Analytical samples were collected at the time when experimental jars and plastic bags were opened for determine silage characteristics. All samples were prepared for analysis by extracting homogenized $50 \mathrm{gm}$ (wet material) with $500 \mathrm{ml}$ distilled water for 10 minutes in a warming blender (Waldo and Schultz, 1956) the homogenate was filtered through four-layer cheese cloth. The filtrate was used to determine $\mathrm{pH}$ directly using a digital $\mathrm{pH}$ meter. Ammonia nitrogen $\left(\mathrm{NH}_{3}-\mathrm{N}\right)$ was determined according to AOAC (2016). The acetic, butyric and lactic acids were determined by the distillation method as reported by Research Institute for cattle feeding at Hoorn, Holland (1961) as described by Nowar (1969).

Rumen fluid samples were taken from rams using a stomach tube at 0 time (before feeding), $2 \mathrm{~h}$ and $4 \mathrm{~h}$ post feeding. These samples were filtered through three layers of surgical gauze without squeezing. Ruminal $\mathrm{pH}$ 
was immediately estimated by digital $\mathrm{pH}$ meter. Rumen ammonia-N was determined according to Conway (1957). Total volatile fatty acids (TVFAs) were measured by the steam distillation method as described by Warner (1964). Microbial protein was determined by the sodium tungstate method according to Shultz and Shultz (1970).

All data were subjected to analysis was performed using the General linear Models (GLM) procedure of the SPSS 24. Mean differences were compared using Duncan multiple range test (Duncan, 1955). Data were analyzed using the following mathematical model:

Yij $=\mu+\mathrm{Ti}+$ eij

$\mathrm{Yij}=$ Individual observation, $\mu=$ overall mean,

$\mathrm{Ti}=$ effect of the $\mathrm{i}^{\text {th }}$ treatments and

eij $=$ Random residual error

\section{RESULTS AND DISCUSSION}

Chemical analysis of ingredients is presented in Table (1). DM contents of potato (18.18\%) and Sweet potato $(20.08 \%)$ were higher than turnip $(7.50 \%)$. OM of potato $(81.85 \%)$ and sweet potato $(79.58 \%)$ were lower than turnip (88.53\%). CP of potato $(13.31 \%)$ and turnip (13.46\%) was higher than sweet potato (10.46\%). $\mathrm{DM} \%$ of potato tubers lie within the range values obtained by Leonel et al. (2017). The DM\% of sweet potato roots lie within the range values obtained by Samy et al. (2014). The CP\% of potato was nearly with $\mathrm{CP}$ obtained by Charmley et al. (2006). The CP\% in sweet potato roots lie within the range values obtained by Samy et al. (2014). The CP\% of turnip roots lie within the data obtained by Ali et al. (2014), Altinok and Karakaya (2003) and Jacobs et al. (2001).

Table (1): Chemical composition of potato tubers, sweet potato roots turnip roots, CFM, rice straw and wheat straw On DM basis $(\%)$

\begin{tabular}{lcccccc}
\hline Items & $\begin{array}{c}\text { Potato } \\
\text { tubers }\end{array}$ & $\begin{array}{c}\text { Sweet potato } \\
\text { roots }\end{array}$ & $\begin{array}{c}\text { Turnip } \\
\text { roots }\end{array}$ & CFM* & RS & WS \\
\hline DM & 18.18 & 20.08 & 7.50 & 92.21 & 89.36 & 90.70 \\
OM & 81.85 & 79.58 & 88.53 & 91.65 & 86.25 & 87.21 \\
CP & 13.31 & 10.46 & 13.46 & 16.81 & 3.25 & 3.42 \\
EE & 1.10 & 0.80 & 0.93 & 3.80 & 1.86 & 2.10 \\
CF & 7.10 & 7.02 & 14.67 & 12.38 & 38.23 & 35.39 \\
NFE & 60.34 & 61.30 & 59.47 & 58.66 & 42.91 & 46.30 \\
Ash & 18.15 & 20.42 & 11.47 & 8.35 & 13.75 & 12.79 \\
\hline
\end{tabular}

CFM: concentrate feed mixture, RS: rice straw, WS: wheat straw

* CFM was formulated from $24 \%$ Sunflower meal, $15 \%$ wheat bran, $55 \%$ yellow corn, $3 \%$ molasses, $2 \%$ lime stone and $1 \%$ common salt

Chemical analysis of silages of Potato, Sweet potato and turnip with RS or WS with or without urea additives ensiling in jars in experiment1 is presented in Table (2). DM of potato silage ranged from 34.31 to $35.68 \%$, DM of sweet potato silage ranged from 34.39 to $35.73 \%$ and DM of turnip silage ranged from 31.57 to $37.71 \%$. The chemical composition explained that $\mathrm{OM} \%$ of potato silage and sweet potato silage was lower than OM\% in turnip silage with or without urea. The $\mathrm{CP} \%$ of potato silage was higher than sweet potato silage, and $\mathrm{CP} \%$ of sweet potato silage was higher than turnip silage with or without urea. As expected, the $\mathrm{CP} \%$ in all silages was increased with increasing urea levels. However, the chemical composition values of silages with RS or WS was nearly similar.

Chemical composition of silages in experiment 2 of potato, sweet potato and turnip with RS in plastic bags which fed by rams as shown in Table (4) explained that DM content of potato silage, sweet potato silage and turnip silage was $36.13,38.14$ and $32.34 \%$, respectively without urea additives and $36.39,37.44$ and $30.19 \%$, respectively with $0.5 \%$ urea. The OM content of potato silage and sweet potato silage was lower than turnip silage with or without urea. The CP content of potato silage and sweet potato silage was slightly higher than turnip silage without urea. The CP content of silages with $0.5 \%$ urea was higher than silages without urea. The EE and NFE of potato silage and sweet potato silage were lower than turnip silage while CF and ash of turnip silage was lower than potato silage and sweet potato silage with or without urea.

Fermentation characteristics of silages in jars as presented in Table (3) explained that $\mathrm{pH}$ values ranged from 3.8 to 4.2 of all treatments with no significant differences among all silages. Ammonia-N values as $\mathrm{g} / 100 \mathrm{~g}$ DM in silages were increased with increasing urea levels. Ammonia-N in potato silage with rice straw significantly $(\mathrm{P}<0.05)$ increased from 0.23 without urea to $0.32 \mathrm{~g} / 100 \mathrm{~g}$ DM with $1 \%$ urea. Ammonia-N in sweet potato silage significantly $(\mathrm{P}<0.05)$ increased from 0.21 without urea to $0.32 \mathrm{~g} / 100 \mathrm{~g} \mathrm{DM}$ with $1 \%$ urea. Ammonia-N in turnip silage significantly $(\mathrm{P}<0.05)$ increased from 0.20 without urea to $0.32 \mathrm{~g} / 100 \mathrm{~g} D \mathrm{DM}$ with $1 \%$ urea. Ammonia-N \% of total $\mathrm{N}$ of sweet potato silage and turnip silage with rice straw with $1 \%$ urea was significantly $(\mathrm{P}<0.05)$ higher than all silages. Acetic acid of sweet potato was significantly $(\mathrm{P}<0.05)$ higher than potato and turnip silages without urea while turnip silage was significantly $(\mathrm{P}<0.05)$ higher than potato and sweet potato silages with $1 \%$ urea. Butyric acid and lactic acid of turnip silages with rice straw without or with $1 \%$ urea was significantly $(\mathrm{P}<0.05)$ higher than potato and sweet potato silages. The effect of urea additives on lactic acid was not clear. 
Fermentation characteristics of silages in plastic bags for rams feeding as shown in Table (4) explained that $\mathrm{pH}$ values in silages of potato, sweet potato and turnip were nearly similar. The $\mathrm{pH}$ values with urea were slightly higher than without urea. Ammonia-N (\% of total $\mathrm{N}$ ) of potato silage with or without urea was significantly $(\mathrm{P}<0.05)$ higher than all silages. Butyric acid in turnip silage with $0.5 \%$ urea was significantly $(\mathrm{P}<0.05)$ higher than all silages. Acetic acid and lactic acid in turnip silage with or without urea were significantly $(\mathrm{P}<0.05)$ higher than all silages.

Table (2): Chemical composition (\% on DM basis) of experimental silages of potato, sweet potato and turnip with rice straw or wheat straw with or without urea ensiling in jars

\begin{tabular}{|c|c|c|c|c|c|c|c|c|c|}
\hline \multirow[t]{2}{*}{ Items } & $\begin{array}{l}\text { Potato } \\
\text { silage }\end{array}$ & $\begin{array}{l}\text { Sweet } \\
\text { potato } \\
\text { silage }\end{array}$ & $\underset{\text { silage }}{\text { Turnip }}$ & $\begin{array}{l}\text { Potato } \\
\text { silage }\end{array}$ & $\begin{array}{l}\text { Sweet } \\
\text { potato } \\
\text { silage }\end{array}$ & $\underset{\text { silage }}{\text { Turnip }}$ & $\begin{array}{l}\text { Potato } \\
\text { silage }\end{array}$ & $\begin{array}{l}\text { Sweet } \\
\text { potato } \\
\text { silage }\end{array}$ & $\underset{\text { silage }}{\text { Turnip }}$ \\
\hline & \multicolumn{3}{|c|}{ Without urea } & \multicolumn{3}{|c|}{ With $0.5 \%$ urea } & \multicolumn{3}{|c|}{ With $1 \%$ urea } \\
\hline \multicolumn{10}{|c|}{ With rice straw } \\
\hline DM & 34.31 & 34.94 & 33.62 & 35.11 & 35.04 & 32.40 & 35.12 & 35.23 & 31.57 \\
\hline OM & 83.83 & 82.41 & 88.94 & 83.91 & 82.17 & 88.22 & 83.63 & 81.98 & 87.59 \\
\hline $\mathbf{C P}$ & 11.66 & 10.02 & 9.52 & 13.04 & 11.40 & 10.90 & 14.44 & 12.80 & 12.30 \\
\hline $\mathbf{E E}$ & 1.25 & 1.37 & 3.87 & 1.35 & 1.31 & 3.40 & 1.48 & 1.70 & 3.17 \\
\hline $\mathbf{C F}$ & 30.55 & 31.25 & 24.06 & 28.48 & 27.87 & 25.03 & 29.40 & 28.40 & 26.61 \\
\hline NFE & 40.37 & 39.77 & 51.49 & 41.04 & 41.59 & 48.89 & 38.31 & 39.08 & 45.54 \\
\hline Ash & 16.17 & 17.59 & 11.06 & 16.09 & 17.83 & 11.78 & 16.37 & 18.02 & 12.41 \\
\hline \multicolumn{10}{|c|}{ With wheat straw } \\
\hline DM & 34.52 & 34.63 & 37.71 & 34.86 & 34.39 & 34.68 & 35.68 & 35.73 & 33.06 \\
\hline OM & 84.43 & 82.75 & 89.48 & 84.29 & 82.33 & 89.49 & 84.38 & 82.72 & 88.67 \\
\hline $\mathbf{C P}$ & 11.33 & 10.52 & 9.78 & 12.70 & 11.90 & 11.18 & 14.10 & 13.30 & 12.58 \\
\hline EE & 1.22 & 1.40 & 2.84 & 1.09 & 1.74 & 2.45 & 1.74 & 1.96 & 3.63 \\
\hline CF & 28.04 & 29.25 & 25.54 & 24.42 & 23.85 & 22.66 & 28.34 & 29.85 & 26.92 \\
\hline NFE & 43.84 & 41.58 & 51.32 & 46.08 & 44.84 & 53.20 & 40.20 & 37.61 & 45.54 \\
\hline Ash & 15.57 & 17.25 & 10.52 & 15.71 & 17.67 & 10.51 & 15.63 & 17.28 & 11.33 \\
\hline
\end{tabular}

Table (3): Silage fermentation characteristics of different silages with rice straw or wheat straw with or without urea in jars

\begin{tabular}{|c|c|c|c|c|c|c|c|c|c|}
\hline Items & $\begin{array}{l}\text { Potato } \\
\text { silage }\end{array}$ & $\begin{array}{c}\text { Sweet } \\
\text { potato } \\
\text { silage } \\
\end{array}$ & $\begin{array}{l}\text { Turnip } \\
\text { silage }\end{array}$ & $\begin{array}{l}\text { Potato } \\
\text { silage }\end{array}$ & $\begin{array}{c}\text { Sweet } \\
\text { potato } \\
\text { silage } \\
\end{array}$ & $\begin{array}{l}\text { Turnip } \\
\text { silage }\end{array}$ & $\begin{array}{l}\text { Potato } \\
\text { silage }\end{array}$ & $\begin{array}{l}\text { Sweet } \\
\text { potato } \\
\text { silage } \\
\end{array}$ & $\begin{array}{l}\text { Turnip } \\
\text { silage }\end{array}$ \\
\hline & \multicolumn{3}{|c|}{ Without urea } & \multicolumn{3}{|c|}{ With $0.5 \%$ urea } & \multicolumn{3}{|c|}{ With $1 \%$ urea } \\
\hline & \multicolumn{9}{|c|}{ With rice straw } \\
\hline pH value & $\begin{array}{c}4.10^{\mathrm{a}} \pm \\
0.06\end{array}$ & $\begin{array}{c}3.80^{\mathrm{b}} \pm \\
0.04\end{array}$ & $\begin{array}{c}4.13^{\mathrm{a}} \pm \\
0.08\end{array}$ & $\begin{array}{c}4.17^{\mathrm{a}} \pm \\
0.02\end{array}$ & $\begin{array}{c}4.20^{\mathrm{a}} \pm \\
0.06\end{array}$ & $\begin{array}{c}4.00^{\mathrm{b}} \pm \\
0.04\end{array}$ & $\begin{array}{c}4.20^{\mathrm{a}} \pm \\
0.06\end{array}$ & $\begin{array}{c}4.20^{\mathrm{a}} \pm \\
0.06\end{array}$ & $\begin{array}{c}4.10^{\mathrm{a}} \pm \\
0.06\end{array}$ \\
\hline $\begin{array}{l}\text { Ammonia-N } \\
(\mathrm{g} / \mathbf{1 0 0 g D M})\end{array}$ & $\begin{array}{c}0.23^{\mathrm{a}} \pm \\
0.01\end{array}$ & $\begin{array}{c}0.21^{\mathrm{b}} \pm \\
0.01\end{array}$ & $\begin{array}{c}0.20^{\mathrm{b}} \pm \\
0.01\end{array}$ & $\begin{array}{c}0.29^{\mathrm{a}} \pm \\
0.01\end{array}$ & $\begin{array}{c}0.24^{\mathrm{b}} \pm \\
0.01\end{array}$ & $\begin{array}{c}0.21^{\mathrm{c}} \pm \\
0.01\end{array}$ & $\begin{array}{c}0.32^{\mathrm{a}} \pm \\
0.01\end{array}$ & $\begin{array}{c}0.32^{\mathrm{a}} \pm \\
0.01\end{array}$ & $\begin{array}{c}0.32^{\mathrm{a}} \pm \\
0.01\end{array}$ \\
\hline $\begin{array}{l}\text { Ammonia-N (\% } \\
\text { of total } N)\end{array}$ & $\begin{array}{c}12.25^{\mathrm{b}} \pm \\
0.01\end{array}$ & $\begin{array}{c}13.00^{\mathrm{a}} \pm \\
0.01\end{array}$ & $\begin{array}{c}13.12^{\mathrm{a}} \pm \\
0.01\end{array}$ & $\begin{array}{c}13.76^{\mathrm{a}} \pm \\
0.01\end{array}$ & $\begin{array}{c}13.14^{\mathrm{a}} \pm \\
0.01\end{array}$ & $\begin{array}{c}11.89^{\mathrm{b}} \pm \\
0.01\end{array}$ & $\begin{array}{c}13.80^{\mathrm{c}} \pm \\
0.01\end{array}$ & $\begin{array}{c}15.52^{\mathrm{b}} \pm \\
0.01\end{array}$ & $\begin{array}{c}16.22^{\mathrm{a}} \pm \\
0.01\end{array}$ \\
\hline $\begin{array}{l}\text { Acetic acid } \\
\text { (g/100g DM) }\end{array}$ & $\begin{array}{c}2.83^{\mathrm{c}} \pm \\
0.01\end{array}$ & $\begin{array}{c}3.22^{\mathrm{a}} \pm \\
0.01\end{array}$ & $\begin{array}{c}3.16^{\mathrm{b}} \pm \\
0.01\end{array}$ & $\begin{array}{c}2.49^{\mathrm{b}} \pm \\
0.05\end{array}$ & $\begin{array}{c}3.05^{\mathrm{a}} \pm \\
0.01\end{array}$ & $\begin{array}{c}3.22^{\mathrm{a}} \pm \\
0.01\end{array}$ & $\begin{array}{c}2.83^{\mathrm{a}} \pm \\
0.01\end{array}$ & $\begin{array}{c}2.47^{\mathrm{c}} \pm \\
0.01\end{array}$ & $\begin{array}{c}3.07^{\mathrm{a}} \pm \\
0.01\end{array}$ \\
\hline $\begin{array}{l}\text { Butyric acid } \\
\text { (g/100g DM) }\end{array}$ & $\begin{array}{c}0.45^{\mathrm{b}} \pm \\
0.01\end{array}$ & $\begin{array}{c}0.50^{\mathrm{b}} \pm \\
0.01\end{array}$ & $\begin{array}{c}0.99^{\mathrm{a}} \pm \\
0.01\end{array}$ & $\begin{array}{c}0.69^{\mathrm{a}} \pm \\
0.03\end{array}$ & $\begin{array}{c}0.34^{\mathrm{c}} \pm \\
0.01\end{array}$ & $\begin{array}{c}0.51^{\mathrm{b}} \pm \\
0.01\end{array}$ & $\begin{array}{c}0.28^{\mathrm{b}} \pm \\
0.01\end{array}$ & $\begin{array}{c}0.64^{\mathrm{a}} \pm \\
0.01\end{array}$ & $\begin{array}{c}0.80^{\mathrm{a}} \pm \\
0.01\end{array}$ \\
\hline $\begin{array}{l}\text { Lactic acid } \\
\text { (g/100g DM) }\end{array}$ & $\begin{array}{c}9.17^{\mathrm{b}} \pm \\
0.01\end{array}$ & $\begin{array}{c}9.05^{\mathrm{b}} \pm \\
0.01\end{array}$ & $\begin{array}{c}9.65^{\mathrm{a}} \pm \\
0.01\end{array}$ & $\begin{array}{c}6.61^{\mathrm{c}} \pm \\
0.01\end{array}$ & $\begin{array}{c}7.06^{\mathrm{b}} \pm \\
0.01\end{array}$ & $\begin{array}{c}7.30^{\mathrm{a}} \pm \\
0.01\end{array}$ & $\begin{array}{c}6.82^{\mathrm{b}} \pm \\
0.01\end{array}$ & $\begin{array}{c}6.31^{\mathrm{c}} \pm \\
0.01\end{array}$ & $\begin{array}{c}8.83^{\mathrm{a}} \pm \\
0.01\end{array}$ \\
\hline \multicolumn{10}{|c|}{ With wheat straw } \\
\hline pH value & $\begin{array}{c}3.80^{\mathrm{a}} \pm \\
0.06\end{array}$ & $\begin{array}{c}3.90^{\mathrm{a}} \pm \\
0.01\end{array}$ & $\begin{array}{c}3.85^{\mathrm{a}} \pm \\
0.02\end{array}$ & $\begin{array}{c}3.85^{\mathrm{a}} \pm \\
0.08\end{array}$ & $\begin{array}{c}3.84^{\mathrm{a}} \pm \\
0.03\end{array}$ & $\begin{array}{c}4.00^{\mathrm{a}} \pm \\
0.06\end{array}$ & $\begin{array}{c}4.00^{\mathrm{a}} \pm \\
0.06\end{array}$ & $\begin{array}{c}4.01^{\mathrm{a}} \pm \\
0.05\end{array}$ & $\begin{array}{c}4.01^{\mathrm{a}} \pm \\
0.05\end{array}$ \\
\hline $\begin{array}{l}\text { Ammonia-N } \\
(\mathrm{g} / 100 \mathrm{~g} \text { DM) }\end{array}$ & $\begin{array}{c}0.26^{\mathrm{a}} \pm \\
0.01\end{array}$ & $\begin{array}{c}0.16^{\mathrm{c}} \pm \\
0.01\end{array}$ & $\begin{array}{c}0.21^{\mathrm{b}} \pm \\
0.01\end{array}$ & $\begin{array}{c}0.26^{\mathrm{a}} \pm \\
0.01\end{array}$ & $\begin{array}{c}0.26^{\mathrm{a}} \pm \\
0.01\end{array}$ & $\begin{array}{c}0.24^{\mathrm{b}} \pm \\
0.01\end{array}$ & $\begin{array}{c}0.31^{\mathrm{a}} \pm \\
0.01\end{array}$ & $\begin{array}{c}0.31^{\mathrm{a}} \pm \\
0.01\end{array}$ & $\begin{array}{c}0.25^{\mathrm{b}} \pm \\
0.01\end{array}$ \\
\hline $\begin{array}{l}\text { Ammonia-N (\% } \\
\text { of total } N)\end{array}$ & $\begin{array}{c}14.32^{\mathrm{a}} \pm \\
0.02\end{array}$ & $\begin{array}{c}9.61^{\mathrm{c}} \pm \\
0.01\end{array}$ & $\begin{array}{c}13.29^{\mathrm{b}} \pm \\
0.02\end{array}$ & $\begin{array}{c}12.65^{\mathrm{b}} \pm \\
0.01\end{array}$ & $\begin{array}{c}13.68^{\mathrm{a}} \pm \\
0.01\end{array}$ & $\begin{array}{c}13.54^{\mathrm{a}} \pm \\
0.01\end{array}$ & $\begin{array}{c}13.91^{\mathrm{b} \pm} \\
0.01\end{array}$ & $\begin{array}{c}14.73^{\mathrm{a}} \pm \\
0.01\end{array}$ & $\begin{array}{c}12.62^{\mathrm{c}} \pm \\
0.01\end{array}$ \\
\hline $\begin{array}{l}\text { Acetic acid } \\
\text { (g/100g DM) }\end{array}$ & $\begin{array}{c}2.63^{\mathrm{b}} \pm \\
0.01\end{array}$ & $\begin{array}{c}3.13^{\mathrm{a}} \pm \\
0.04\end{array}$ & $\begin{array}{c}2.74^{\mathrm{b}} \pm \\
0.03\end{array}$ & $\begin{array}{c}2.99^{\mathrm{b}} \pm \\
0.05\end{array}$ & $\begin{array}{c}3.43^{\mathrm{a}} \pm \\
0.01\end{array}$ & $\begin{array}{c}2.97^{\mathrm{b}} \pm \\
0.02\end{array}$ & $\begin{array}{c}2.73^{\mathrm{b}} \pm \\
0.03\end{array}$ & $\begin{array}{c}3.09^{\mathrm{a}} \pm \\
0.02\end{array}$ & $\begin{array}{c}3.52^{\mathrm{a}} \pm \\
0.03\end{array}$ \\
\hline $\begin{array}{l}\text { Butyric acid } \\
\text { (g/100g DM) }\end{array}$ & $\begin{array}{c}0.39^{\mathrm{c}} \pm \\
0.01\end{array}$ & $\begin{array}{c}1.26^{\mathrm{a}} \pm \\
0.01\end{array}$ & $\begin{array}{c}1.14^{\mathrm{b}} \pm \\
0.01\end{array}$ & $\begin{array}{c}1.34^{\mathrm{a}} \pm \\
0.03\end{array}$ & $\begin{array}{c}1.07^{\mathrm{b}} \pm \\
0.01\end{array}$ & $\begin{array}{c}0.41^{\mathrm{c}} \pm \\
0.01\end{array}$ & $\begin{array}{c}1.00^{\mathrm{a}} \pm \\
0.01\end{array}$ & $\begin{array}{c}0.50^{\mathrm{c}} \pm \\
0.01\end{array}$ & $\begin{array}{c}0.70^{\mathrm{b}} \pm \\
0.01\end{array}$ \\
\hline $\begin{array}{l}\text { Lactic acid } \\
\text { (g/100g DM) }\end{array}$ & $\begin{array}{c}8.33^{\mathrm{b}} \pm \\
0.01\end{array}$ & $\begin{array}{c}9.30^{\mathrm{a}} \pm \\
0.01\end{array}$ & $\begin{array}{c}6.85^{\mathrm{c}} \pm \\
0.01\end{array}$ & $\begin{array}{c}7.70^{\mathrm{a}} \pm \\
0.03\end{array}$ & $\begin{array}{c}6.97^{\mathrm{b}} \pm \\
0.01\end{array}$ & $\begin{array}{c}6.80^{\mathrm{b}} \pm \\
0.01\end{array}$ & $\begin{array}{c}7.79^{\mathrm{a}} \pm \\
0.01\end{array}$ & $\begin{array}{c}6.49^{\mathrm{b}} \pm \\
0.01\end{array}$ & $\begin{array}{c}7.55^{\mathrm{a}} \pm \\
0.01\end{array}$ \\
\hline
\end{tabular}


Table (4): Chemical composition and silage fermentation characteristics of silages of potato, sweet potato and turnip with rice straw with and without urea fed by rams

\begin{tabular}{|c|c|c|c|c|c|c|}
\hline \multirow[t]{2}{*}{ Items } & $\begin{array}{l}\text { Potato } \\
\text { silage }\end{array}$ & $\begin{array}{c}\text { Sweet potato } \\
\text { silage }\end{array}$ & $\begin{array}{l}\text { Turnip } \\
\text { silage }\end{array}$ & $\begin{array}{l}\text { Potato } \\
\text { silage }\end{array}$ & $\begin{array}{c}\text { Sweet potato } \\
\text { silage }\end{array}$ & $\begin{array}{l}\text { Turnip } \\
\text { silage }\end{array}$ \\
\hline & \multicolumn{3}{|c|}{ Without urea } & \multicolumn{3}{|c|}{ With $0.5 \%$ urea } \\
\hline \multicolumn{7}{|c|}{ Chemical composition (\%) on DM basis } \\
\hline $\mathbf{D M}$ & 36.13 & 38.14 & 32.34 & 36.39 & 37.44 & 30.19 \\
\hline OM & 84.65 & 83.88 & 88.50 & 84.48 & 83.31 & 87.36 \\
\hline $\mathbf{C P}$ & 10.99 & 10.39 & 9.71 & 12.37 & 11.81 & 11.13 \\
\hline $\mathbf{E E}$ & 1.94 & 1.84 & 3.09 & 1.59 & 1.68 & 2.65 \\
\hline CF & 31.44 & 29.23 & 28.66 & 30.78 & 29.81 & 28.62 \\
\hline NFE & 40.28 & 42.42 & 47.04 & 39.74 & 40.01 & 44.96 \\
\hline Ash & 15.35 & 16.12 & 11.50 & 15.52 & 16.69 & 12.64 \\
\hline \multicolumn{7}{|c|}{ Silage fermentation characteristics } \\
\hline pH value & $3.94^{\mathrm{a}} \pm 0.01$ & $3.98^{\mathrm{a}} \pm 0.01$ & $3.86^{\mathrm{b}} \pm 0.01$ & $4.20^{\mathrm{a}} \pm 0.06$ & $4.10^{\mathrm{a}} \pm 0.06$ & $4.20^{\mathrm{a}} \pm 0.06$ \\
\hline $\begin{array}{l}\text { Ammonia-N (\% } \\
\text { of total } N)\end{array}$ & $14.10^{\mathrm{a}} \pm 0.01$ & $12.37^{\mathrm{c}} \pm 0.01$ & $13.37^{\mathrm{b}} \pm 0.01$ & $13.22^{\mathrm{a}} \pm 0.01$ & $7.92^{c} \pm 0.01$ & $12.50^{\mathrm{b}} \pm 0.01$ \\
\hline $\begin{array}{l}\text { Acetic acid } \\
(\mathrm{g} / 100 \mathrm{~g} D \mathrm{DM})\end{array}$ & $2.89^{b} \pm 0.01$ & $2.36^{\mathrm{c}} \pm 0.01$ & $3.07^{\mathrm{a}} \pm 0.01$ & $2.66^{\mathrm{b}} \pm 0.01$ & $2.66^{\mathrm{b}} \pm 0.01$ & $3.50^{\mathrm{a}} \pm 0.01$ \\
\hline $\begin{array}{l}\text { Butyric acid } \\
\text { (g/100g DM) }\end{array}$ & $0.93^{\mathrm{a}} \pm 0.01$ & $0.05^{c} \pm 0.01$ & $0.27^{b} \pm 0.03$ & $1.16^{\mathrm{b}} \pm 0.01$ & $0.36^{\mathrm{c}} \pm 0.01$ & $1.67^{\mathrm{a}} \pm 0.01$ \\
\hline $\begin{array}{l}\text { Lactic acid } \\
\text { (g/100g DM) }\end{array}$ & $7.95^{\mathrm{a}} \pm 0.01$ & $6.26^{\mathrm{b}} \pm 0.01$ & $8.20^{\mathrm{a}} \pm 0.01$ & $6.47^{\mathrm{b}} \pm 0.01$ & $6.64^{\mathrm{b}} \pm 0.01$ & $8.10^{\mathrm{a}} \pm 0.01$ \\
\hline
\end{tabular}

The DM percent of silages in this study was nearly similar with Mutavhatsindi et al. (2018) (35.2\%), Sadri et al. (2018) (32.2\%), Babaeinasab et al. (2015) (32.2$36.0 \%)$, Hough et al. (1994) (38.2\%) and Hart and Horn (1987) (32.2\%). However, the chemical composition of silage is affected with difference of silage components. Pen et al. (2006) found that CP was $14.40 \%$ in silage containing potato by-products. Hadgu et al. (2015) found that $\mathrm{CP}$ in sweet potato silage ranged from 10.9 to 16.2\%. Hart and Horn (1987) found that CP ranged from 7.7 to $10.5 \%$ in turnip-wheat straw silage.

Silage fermentation characteristics in this study explained that $\mathrm{pH}$ as important indicator for silage fermentation quality ranged from 3.80 to 4.20 of potato, sweet potato and turnip silages. These values agree with those obtained by Rui-rui et al. (2018), Sugimoto et al. (2010), Hough et al. (1994), Hadgu et al. (2015), Babaeinasab et al. (2015) and Nkosi and Meeske (2010). Sugimoto et al. (2007) noticed that $\mathrm{pH}$ value of potato pulp silage without urea was less than ureatreated potato pulp silage. Hart and Horn (1987) noticed that $\mathrm{pH}$ value of silage containing turnip and wheat straw was lower than that silage containing turnip and ammoniated wheat straw. Generally, good quality silage is containing $\mathrm{pH}$ under 4.8 (Alberta Agriculture Food and Rural Development 2004). Ammonia-N in this study ranged from 0.15 to $0.32 \mathrm{~g} / 100 \mathrm{~g}$ DM. These values agree with those obtained by Kleinschmit and Kung (2006) and Hart and Horn (1987). Ammonia-N\% of total $\mathrm{N}$ in this study was less than $15 \%$ except sweet potato and turnip silages containing RS with $1 \%$ urea which was 15.52 and $16.22 \%$. These results agreed with Rigueira et al. (2013) who found that ammonia-N ranged from 10 to 20.7 from total $\mathrm{N}$ and Nicholson and Macleo (1966) who found that ammonia $\mathrm{N}$ in various silages ranged from 11.9 to $16.5 \%$ of total $\mathrm{N}$. On the other hand, ammonia- $\mathrm{N} \%$ of total $\mathrm{N}$ in this study was higher than that obtained by Rui-rui et al. (2018), Babainasab et al. (2015) and Giang et al. (2004). However, Kung et al. (2018) mentioned that $\mathrm{NH}_{3}-\mathrm{N}$ usually less than $15 \%$ of total $\mathrm{N}$ of silage and Mahanna (1994) mentioned that silages containing $10-15 \%$ ammonia- $\mathrm{N}$ of total $\mathrm{N}$ are considered of good quality silage. Generally, ammonia-N from silage with available energy in the rumen is using by rumen microorganisms for synthesis microbial protein. Acetic acid in silage in this study ranged from 2.36 to 3.52 $\mathrm{g} / 100 \mathrm{~g}$ DM. These values agree with those obtained by Giang et al. (2004) and Hart and Horn (1987) and were higher than that obtained by Rui-rui et al. (2018), Nkosi and Meeske (2010) and Okine et al. (2007). However, Ruiz et al. (1981) mentioned that there are no norms indicating optimum or maximum values of acetic acid in good quality silages. Generally, the effect of high acetic acid concentration on intake of silage remains unclear (Alberta Agriculture Food and Rural Development 2004). Kung et al. (2018) explained that acetic acid of silage absorbed from the rumen and can be used for energy source in ruminants. Butyric acid in this study was less than $1 \%$ of DM in all silages with rice straw without urea. Similar results were showed by Kung et al. (2018) and Nicholson and Macleo (1966). On the other side, Butyric acid in silage with urea was higher than that without urea. The same trend was showed by Ruiz et al. (1981) who found that butyric acid production was increased with increasing urea levels in sweet potato silage and Hart and Horn (1987) who found that butyric acid of silage containing turnip and ammoniated wheat straw was higher than silage of turnip and wheat straw without ammonia. Giang et al. (2004) found that butyric acid ranged from 0.33 to 0.47 $\mathrm{g} / \mathrm{kg}$ DM of sweet potato silage while Ruiz et al. (1981) found that butyric acid ranged from 1.14 to $3.28 \%$ on DM basis of sweet potato silage. Lactic acid in silages 
of potato, sweet potato and turnip in this study ranged from 6.31 to $9.65 \mathrm{~g} / 100 \mathrm{~g}$ DM (6.31 to $9.65 \%)$. Similar values were found by Mutavhatsindi et al. (2018), Okine et al. (2005), Abo-Donia et al. (2004) and Hart and Horn (1987). However, these values lie within the normal data of good quality silage as reported by Zobell et al. (2005) who stated that the high levels of lactic acid concentration between 3 - 14\% DM characterize good quality silage. Also, McDonald et al. (2010) mentioned that the lactic acid contents generally lie in the range $8-12 \%$ of silage DM. The effect of urea on lactic acid was fluctuated. The same trend was showed by Ruiz et al. (1981). Generally, lactic acid from silage is converted to propionic acid in the rumen under normal feeding conditions (Kung et al., 2018).
The values of DM intake (Table 5) as $\mathrm{g} / \mathrm{head} / \mathrm{day}$, $\%$ of $\mathrm{LBW}$ and $\mathrm{g} / \mathrm{kg} \mathrm{W}^{0.75}$ of ration $\mathrm{A}$ (control) (containing $\mathrm{CFM}+\mathrm{RS}$ ) were significantly $(\mathrm{P}<0.05)$ higher than all rations (containing CFM + silages). The differences of DM intake as \% of LBW and $\mathrm{g} / \mathrm{kg} \mathrm{W}^{0.75}$ among all rations containing silages $(\mathrm{B}, \mathrm{C}, \mathrm{D}, \mathrm{E}, \mathrm{F}$ and $\mathrm{G})$ were not significant and silages intakes were nearly similar. The differences of DM intake among the rations containing silages with or without urea were not significant. The same trend was showed by Sugimoto et al. (2007) who noticed that treating of potato pulp silage with urea did not affect the DM intake. However, Sadri et al. (2018) found that the daily intake of DM was not significantly affected by different levels of potato-wheat straw silage in the rations.

Table (5): Intake, digestion coefficients and nutritive values of experimental rations by rams

\begin{tabular}{|c|c|c|c|c|c|c|c|}
\hline \multirow{2}{*}{ Items } & \multirow{2}{*}{ Ration A } & \multicolumn{3}{|c|}{ Without urea additives } & \multicolumn{3}{|c|}{ With $0.5 \%$ urea additives } \\
\hline & & Ration B & Ration C & Ration D & Ration E & Ration F & Ration G \\
\hline \multicolumn{8}{|c|}{ DM intake } \\
\hline CFM, g/h/d & 885 & 538 & 547 & 541 & 532 & 553 & 518 \\
\hline $\mathbf{R S}, \mathbf{g} / \mathbf{h} / \mathbf{d}$ & 411 & - & - & - & - & - & - \\
\hline Silage, $g / h / d$ & - & 355 & 407 & 341 & 360 & 399 & 308 \\
\hline Total, g/h/d & $1296^{\mathrm{a}}$ & $893^{\mathrm{c}}$ & $954^{\mathrm{b}}$ & $882^{\mathrm{c}}$ & $892^{\mathrm{c}}$ & $952^{\mathrm{b}}$ & $826^{\mathrm{d}}$ \\
\hline $\begin{array}{l}\text { Total, \% of } \\
\text { LBW }\end{array}$ & $2.43^{\mathrm{a}}$ & $1.86^{\mathrm{b}}$ & $1.97^{\mathrm{b}}$ & $1.85^{\mathrm{b}}$ & $1.87^{\mathrm{b}}$ & $1.96^{\mathrm{b}}$ & $1.74^{\mathrm{b}}$ \\
\hline $\begin{array}{l}\text { Total, g/kg } \\
\mathbf{w}^{0.75}\end{array}$ & $65.72^{\mathrm{a}}$ & $48.92^{\mathrm{b}}$ & $51.87^{\mathrm{b}}$ & $48.61^{\mathrm{b}}$ & $49.03^{\mathrm{b}}$ & $51.82^{\mathrm{b}}$ & $45.55^{\mathrm{b}}$ \\
\hline \multicolumn{8}{|c|}{ Digestion coefficients $\%$} \\
\hline DM & $\begin{array}{c}62.67^{\mathrm{a}} \pm \\
0.47\end{array}$ & $\begin{array}{c}56.94^{\mathrm{b}} \pm \\
0.25\end{array}$ & $\begin{array}{c}54.09^{\mathrm{b}} \pm \\
0.73\end{array}$ & $\begin{array}{c}53.38^{\mathrm{b}} \pm \\
2.75\end{array}$ & $\begin{array}{c}54.98^{\mathrm{b}} \pm \\
1.11\end{array}$ & $\begin{array}{c}54.45^{\mathrm{b}} \pm \\
0.71\end{array}$ & $\begin{array}{c}52.88^{\mathrm{b}} \pm \\
1.02\end{array}$ \\
\hline $\mathbf{O M}$ & $\begin{array}{c}63.32^{\mathrm{a}} \pm \\
0.47\end{array}$ & $\begin{array}{c}59.03^{\mathrm{b}} \pm \\
0.24\end{array}$ & $\begin{array}{c}54.69^{c} \pm \\
0.71\end{array}$ & $\begin{array}{c}56.42^{\mathrm{bc}} \pm \\
2.57\end{array}$ & $\begin{array}{c}56.66^{\mathrm{bc}} \pm \\
1.08\end{array}$ & $\begin{array}{c}55.27^{\mathrm{bc}} \pm \\
0.69\end{array}$ & $\begin{array}{c}55.57^{\mathrm{bc}} \pm \\
0.96\end{array}$ \\
\hline $\mathbf{C P}$ & $\begin{array}{c}64.57^{\mathrm{a}} \pm \\
0.46\end{array}$ & $\begin{array}{c}64.48^{\mathrm{a}} \pm \\
0.20\end{array}$ & $\begin{array}{c}60.64^{\mathrm{abc}} \pm \\
0.06\end{array}$ & $\begin{array}{c}55.27^{\mathrm{c}} \pm \\
2.69\end{array}$ & $\begin{array}{c}63.62^{\mathrm{ab}} \pm \\
0.94\end{array}$ & $\begin{array}{c}58.61^{\mathrm{bc}} \pm \\
0.64\end{array}$ & $\begin{array}{c}60.88^{\mathrm{abc}} \pm \\
0.86\end{array}$ \\
\hline CF & $\begin{array}{c}58.87^{\mathrm{a}} \pm \\
0.51\end{array}$ & $\begin{array}{c}51.62^{\mathrm{b}} \pm \\
0.49\end{array}$ & $\begin{array}{c}51.71^{\mathrm{b}} \pm \\
0.97\end{array}$ & $\begin{array}{c}47.30^{\mathrm{bc}} \pm \\
2.99\end{array}$ & $\begin{array}{c}49.17^{\mathrm{bc}} \pm \\
1.23\end{array}$ & $\begin{array}{c}48.14^{\mathrm{bc}} \pm \\
0.81\end{array}$ & $\begin{array}{c}45.53^{\mathrm{c}} \pm \\
1.16\end{array}$ \\
\hline $\mathbf{E E}$ & $\begin{array}{c}89.10^{\mathrm{a}} \pm \\
0.14\end{array}$ & $\begin{array}{c}86.97^{\mathrm{bc}} \pm \\
0.08\end{array}$ & $\begin{array}{c}88.11^{\mathrm{ab}} \pm \\
0.17\end{array}$ & $\begin{array}{c}87.85^{\mathrm{b}} \pm \\
0.72\end{array}$ & $\begin{array}{c}85.09^{\mathrm{d}} \pm \\
0.42\end{array}$ & $\begin{array}{c}82.79^{\mathrm{e}} \pm \\
0.27\end{array}$ & $\begin{array}{c}86.51^{\mathrm{c}} \pm \\
0.29\end{array}$ \\
\hline NFE & $\begin{array}{c}63.20^{\mathrm{a}} \pm \\
0.47\end{array}$ & $\begin{array}{c}58.67^{\mathrm{ab}} \pm \\
0.23\end{array}$ & $\begin{array}{c}52.28^{\mathrm{c}} \pm \\
0.70\end{array}$ & $\begin{array}{c}57.80^{\mathrm{ab}} \pm \\
2.51\end{array}$ & $\begin{array}{c}55.84^{\mathrm{b}} \pm \\
1.15\end{array}$ & $\begin{array}{c}55.49^{\mathrm{b}} \pm \\
0.69\end{array}$ & $\begin{array}{c}55.53^{\mathrm{b}} \pm \\
0.97\end{array}$ \\
\hline \multicolumn{8}{|c|}{ Nutritive values $\%$} \\
\hline TDN & $\begin{array}{c}60.49^{\mathrm{a}} \pm \\
0.43\end{array}$ & $\begin{array}{c}55.83^{\mathrm{b}} \pm \\
0.21\end{array}$ & $\begin{array}{c}51.61^{\mathrm{c}} \pm \\
0.62\end{array}$ & $\begin{array}{c}54.91^{\mathrm{bc}} \pm \\
2.36\end{array}$ & $\begin{array}{c}53.44^{\mathrm{bc}} \pm \\
0.99\end{array}$ & $\begin{array}{c}51.74^{\mathrm{c}} \pm \\
0.62\end{array}$ & $\begin{array}{c}53.73^{\mathrm{bc}} \pm \\
0.88\end{array}$ \\
\hline DCP & $\begin{array}{c}8.08^{\mathrm{de}} \pm \\
0.06\end{array}$ & $\begin{array}{c}9.37^{\mathrm{a}} \pm \\
0.04\end{array}$ & $\begin{array}{c}8.55^{\mathrm{cd}} \pm \\
0.08\end{array}$ & $\begin{array}{c}7.79^{\mathrm{e}} \pm \\
0.39\end{array}$ & $\begin{array}{c}9.58^{\mathrm{a}} \pm \\
0.16\end{array}$ & $\begin{array}{c}8.62^{\text {cd }} \pm \\
0.10\end{array}$ & $\begin{array}{c}8.99^{\mathrm{bc}} \pm \\
0.13\end{array}$ \\
\hline
\end{tabular}

,b,c,d means in the same row with different superscripts are significantly different $(\mathrm{P}<0.05)$

Digestion coefficients of experimental rations as shown in Table (5) explained that digestion coefficients of $\mathrm{DM}$ and $\mathrm{OM} \%$ of ration A (control) were significantly $(\mathrm{P}<0.05)$ higher than all rations and the differences among other rations containing silages were not significant. The differences of $\mathrm{CP}$ digestibility among rations $\mathrm{C}, \mathrm{D}, \mathrm{E}$ and $\mathrm{G}$ were not significant. Also, the differences of $\mathrm{CP}$ digestibility among rations $\mathrm{A}, \mathrm{B}$, $\mathrm{C}, \mathrm{E}$ and $\mathrm{G}$ were not significant. However, the digestion coefficients of DM of rations containing silages ranged from 52.88 to $56.94 \%$, OM ranged from 54.69 to $59.03 \%$ and CP ranged from 58.61 to $64.8 \%$. Similar values were obtained by Nkosi et al. (2010) who found that digestion coefficients of DM ranged from 49.30 to $59.31 \%$, OM ranged from 48.95 to $59.51 \%$ and $\mathrm{CP}$ ranged from 40.4 to $65.3 \%$ in the rations containing silage potato hash. Sugimoto et al. (2007) found that digestion coefficients of DM, OM and CP were 58.3, 60.1 and $71.90 \%$, respectively of ration containing potato pulp silage while Sugimoto et al. (2010) found that digestion coefficients of DM, OM and $\mathrm{CP}$ were $63.7,65.1$ and $41.8 \%$, respectively of ration containing potato pulp silage. The CF digestibility of ration A was significantly $(\mathrm{P}<0.05)$ higher than other rations while the differences among all rations containing silages were not significant except ration $G$ was significantly 
$(\mathrm{P}<0.05)$ lower than rations $\mathrm{B}$ and $\mathrm{C}$. The NFE digestibility of ration $\mathrm{C}$ was significantly $(\mathrm{P}<0.05)$ lower than other rations. The differences of NFE digestibility among control and rations $\mathrm{B}$ and $\mathrm{D}$ were not significant, also the differences among rations $\mathrm{B}, \mathrm{D}$, $\mathrm{E}, \mathrm{F}$ and $\mathrm{G}$ were not significant. The effect of urea on digestion coefficients of DM, OM, CP and $\mathrm{CF} \%$ was not significant. Similar effect was noticed by Sugimoto et al. (2007). This result was due probably to the slight difference in dietary $\mathrm{CP}$ concentration between the rations contained urea-treated and untreated silages which may be did not affect digestion coefficients. Generally, digestion coefficients are affected by species and age of the animal, chemical composition of feed, level of feeding, preparation of feed, particle size of feed, additives, associative effect of feed components and rate of passage of digesta through the alimentary tract. The TDN in ration A was significantly $(p<0.05)$ higher than all rations containing silages. The TDN of sweet potato silage was significantly $(\mathrm{p}<0.05)$ lower than potato and turnip silages while, the TDN of potato and turnip silages was nearly similar. The differences among rations containing silages with or without urea were not significant. The DCP of rations containing potato silage $(B$ and $E)$ was significantly $(p<0.05)$ higher than all rations. Generally, the TDN was calculated from the percentages and digestion coefficients of $\mathrm{CP}, \mathrm{CF}, \mathrm{EE}$ and NFE in the rations, and DCP was calculated from $\mathrm{CP}$ percent and its digestibility in the rations, therefore the chemical composition and digestion coefficients of these nutrients in the rations was reflected on nutritive values as TDN and DCP.
Rumen fermentation parameters of rams are presented in Table (6). The maximum $\mathrm{pH}$ values were recorded at $0 \mathrm{~h}$ (before feeding) with all groups, then significantly $(\mathrm{P}<0.05)$ decreased at $2 \mathrm{~h}$ then increased at $4 \mathrm{~h}$ post feeding. The same trend was showed by Sugimoto et al. (2007) who found that the maximum ruminal $\mathrm{pH}$ was recorded at 0 time then decreased at $2 \mathrm{~h}$ post feeding and Osman et al. (2007) who found that the maximum ruminal $\mathrm{pH}$ was recorded at 0 time then decreased at $2 \mathrm{~h}$ post feeding then increased at $4 \mathrm{~h}$ post feeding. The differences of ruminal $\mathrm{pH}$ at $2 \mathrm{~h}$ post feeding among rations $\mathrm{A}, \mathrm{B}, \mathrm{C}$ and $\mathrm{D}$ (without urea) were not significant, also the differences among rations $E$, $F$ and $G$ (with urea) were not significant. The differences at $4 \mathrm{~h}$ post feeding of $\mathrm{pH}$ among all rations were not significant. Sugimoto et al. (2007) noticed that urea-treated did not significantly change the $\mathrm{pH}$ in the rumen. Ruminal $\mathrm{pH}$ values in all rations ranged from 5.52 to 7.2. These values are lie within the normal $\mathrm{pH}$ in the rumen as mentioned by Hungate (1966) who mentioned that the normal $\mathrm{pH}$ for normally functioning in the rumen is ranged from 5.5 to 7.3. The lowest values of ruminal $\mathrm{NH}_{3}-\mathrm{N}$ were recorded at $0 \mathrm{~h}$ of all rations, then significantly $(\mathrm{P}<0.05)$ increased at $2 \mathrm{~h}$ and 4h post feeding. Sugimoto et al. (2008) and Osman et al. (2007) noticed that the maximum ruminal $\mathrm{NH}_{3}-\mathrm{N}$ concentration was showed at $2 \mathrm{~h}$ post feeding then decreased. The differences among rations $\mathrm{B}, \mathrm{C}$ and $\mathrm{D}$ (without urea) were not significant. Also, the differences among rations $\mathrm{E}, \mathrm{F}$ and $\mathrm{G}$ (with urea) were not significant at 2 and $4 \mathrm{~h}$ post feeding. Ruminal $\mathrm{NH}_{3}-\mathrm{N}$ of rations containing silages with urea was significantly $(\mathrm{P}<0.05)$ higher than control and rations containing silages without urea.

Table (6): Rumen fermentation parameters in rumen fluid of rams fed experimental rations with or without urea additives

\begin{tabular}{|c|c|c|c|c|c|c|c|c|}
\hline \multirow{2}{*}{ Rumen parameters } & \multirow{2}{*}{ Time } & \multirow{2}{*}{$\begin{array}{c}\text { Ration } \\
\text { A }\end{array}$} & \multicolumn{3}{|c|}{ Without urea additives } & \multicolumn{3}{|c|}{ With $0.5 \%$ urea additives } \\
\hline & & & Ration B & Ration C & Ration D & Ration E & Ration F & Ration G \\
\hline \multirow{3}{*}{ pH } & $\mathbf{0}$ & $\begin{array}{c}6.88^{\mathrm{Ac}} \pm \\
0.04\end{array}$ & $\begin{array}{c}7.09^{\mathrm{Ab}} \pm \\
0.02\end{array}$ & $\begin{array}{c}7.20^{\mathrm{Aa}} \pm \\
0.02\end{array}$ & $\begin{array}{c}7.06^{\mathrm{Ab}} \pm \\
0.03\end{array}$ & $\begin{array}{c}7.08^{\mathrm{Ab}} \pm \\
0.04\end{array}$ & $\begin{array}{c}7.12^{\mathrm{Aab}} \pm \\
0.03\end{array}$ & $\begin{array}{c}7.06^{\mathrm{Ab}} \pm \\
0.04\end{array}$ \\
\hline & 2 & $\begin{array}{c}5.93^{\mathrm{Ca}} \pm \\
0.04\end{array}$ & $\begin{array}{c}5.88^{\mathrm{Ca}} \pm \\
0.02\end{array}$ & $\begin{array}{c}5.82^{\mathrm{Ca}} \pm \\
0.02\end{array}$ & $\begin{array}{c}5.83^{\mathrm{Ca}} \pm \\
0.03\end{array}$ & $\begin{array}{l}5.54^{\mathrm{Cb}} \pm \\
0.05\end{array}$ & $\begin{array}{c}5.52^{\mathrm{Cb}} \pm \\
0.07\end{array}$ & $\begin{array}{c}5.59^{\mathrm{Cb}} \pm \\
0.05\end{array}$ \\
\hline & 4 & $\begin{array}{c}6.50^{\mathrm{Ba}} \pm \\
0.12\end{array}$ & $\begin{array}{c}6.69^{\mathrm{Ba}} \pm \\
0.04\end{array}$ & $\begin{array}{c}6.75^{\mathrm{Ba}} \pm \\
0.12\end{array}$ & $\begin{array}{c}6.64^{\mathrm{Ba}} \pm \\
0.05\end{array}$ & $\begin{array}{c}6.61^{\mathrm{Ba}} \pm \\
0.06\end{array}$ & $\begin{array}{c}6.55^{\mathrm{Ba}} \pm \\
0.06\end{array}$ & $\begin{array}{c}6.61^{\mathrm{Ba}} \pm \\
0.05\end{array}$ \\
\hline \multirow{3}{*}{$\begin{array}{l}\text { Ammonia-N (NH3- } \\
\text { N) }(\mathrm{mg} / 100 \mathrm{ml})\end{array}$} & $\mathbf{0}$ & $\begin{array}{c}26.13^{\mathrm{Ba}} \pm \\
1.56\end{array}$ & $\begin{array}{c}26.60^{\mathrm{Ba}} \pm \\
1.88\end{array}$ & $\begin{array}{c}29.40^{\mathrm{Aa}} \pm \\
3.45\end{array}$ & $\begin{array}{c}30.80^{\mathrm{Ba}} \pm \\
3.15\end{array}$ & $\begin{array}{c}28.93^{\mathrm{Ba}} \pm \\
2.57\end{array}$ & $\begin{array}{c}27.53^{\mathrm{Ba}} \pm \\
1.68\end{array}$ & $\begin{array}{c}31.73^{\mathrm{Ba}} \pm \\
2.47\end{array}$ \\
\hline & 2 & $\begin{array}{l}33.60^{\mathrm{Ad}} \pm \\
1.25\end{array}$ & $\begin{array}{l}37.33^{\mathrm{Acd}} \pm \\
2.25\end{array}$ & $\begin{array}{c}38.27^{\mathrm{Abcd}} \pm \\
2.57\end{array}$ & $\begin{array}{c}40.60^{\mathrm{Abc}} \pm \\
0.96\end{array}$ & $\begin{array}{l}41.07^{\mathrm{Aabc}} \pm \\
2.25\end{array}$ & $\begin{array}{c}42.93^{\mathrm{Aab}} \pm \\
1.18\end{array}$ & $\begin{array}{c}45.27^{\mathrm{Aa}} \pm \\
0.86\end{array}$ \\
\hline & 4 & $\begin{array}{c}37.33^{\mathrm{Ab}} \pm \\
3.00\end{array}$ & $\begin{array}{c}37.80^{\mathrm{Ab}} \pm \\
1.20\end{array}$ & $\begin{array}{l}35.00^{\mathrm{Ab}} \pm \\
2.68\end{array}$ & $\begin{array}{c}37.80^{\mathrm{ABb}} \pm \\
2.48\end{array}$ & $\begin{array}{c}42.93^{\mathrm{Aa}} \pm \\
1.87\end{array}$ & $\begin{array}{l}42.00^{\mathrm{Aa}} \pm \\
2.40\end{array}$ & $\begin{array}{c}43.40^{\mathrm{Aa}} \pm \\
2.37\end{array}$ \\
\hline \multirow{3}{*}{$\begin{array}{l}\text { Total volatile fatty } \\
\text { acids (TVFA's) } \\
\text { (mEq/100ml) }\end{array}$} & $\mathbf{0}$ & $\begin{array}{c}7.50^{\mathrm{Ab}} \pm \\
0.30\end{array}$ & $\begin{array}{c}7.00^{\mathrm{Bb}} \pm \\
0.37\end{array}$ & $\begin{array}{c}6.67^{\mathrm{Ab}} \pm \\
0.75\end{array}$ & $\begin{array}{c}6.25^{\mathrm{Bb}} \pm \\
0.34\end{array}$ & $\begin{array}{c}11.13^{\mathrm{Aa}} \pm \\
0.41\end{array}$ & $\begin{array}{c}11.04^{\mathrm{Aa}} \pm \\
1.12\end{array}$ & $\begin{array}{c}10.67^{\mathrm{Aa}} \pm \\
0.96\end{array}$ \\
\hline & 2 & $\begin{array}{c}8.35^{\mathrm{Ab}} \pm \\
0.33\end{array}$ & $\begin{array}{c}8.00^{\mathrm{ABb}} \pm \\
0.35\end{array}$ & $\begin{array}{c}7.96^{\mathrm{Ab}} \pm \\
0.79\end{array}$ & $\begin{array}{c}8.13^{\mathrm{ABb}} \pm \\
0.61\end{array}$ & $\begin{array}{c}12.13^{\mathrm{Aa}} \pm \\
0.55\end{array}$ & $\begin{array}{c}12.62^{\mathrm{Aa}} \pm \\
0.51\end{array}$ & $\begin{array}{c}11.50^{\mathrm{Aa}} \pm \\
0.43\end{array}$ \\
\hline & 4 & $\begin{array}{c}8.28^{\mathrm{Ab}} \pm \\
0.25\end{array}$ & $\begin{array}{c}8.58^{\mathrm{Aab}} \pm \\
0.69\end{array}$ & $\begin{array}{c}8.92^{\mathrm{Aab}} \pm \\
0.89\end{array}$ & $\begin{array}{c}8.83^{\text {Aab }} \pm \\
0.78\end{array}$ & $\begin{array}{c}12.03^{\mathrm{Aa}} \pm \\
1.50\end{array}$ & $\begin{array}{c}12.27^{\mathrm{Aa}} \pm \\
0.65\end{array}$ & $\begin{array}{c}11.92^{\mathrm{Aab}} \pm \\
0.58\end{array}$ \\
\hline MP(g/100ml) & 4 & $\begin{array}{c}0.72^{\mathrm{a}} \pm \\
0.10\end{array}$ & $\begin{array}{c}0.33^{\mathrm{b}} \pm \\
0.04\end{array}$ & $\begin{array}{c}0.52^{\mathrm{ab}} \pm \\
0.07\end{array}$ & $\begin{array}{c}0.56^{\mathrm{ab}} \pm \\
0.14\end{array}$ & $\begin{array}{c}0.53^{\mathrm{ab}} \pm \\
0.09\end{array}$ & $\begin{array}{c}0.54^{\mathrm{ab}} \pm \\
0.07\end{array}$ & $\begin{array}{c}0.64^{\mathrm{a}} \pm \\
0.09\end{array}$ \\
\hline
\end{tabular}

$\mathrm{A}, \mathrm{B}, \mathrm{C}$ means in the same column with different superscripts are significantly different $(\mathrm{P}<0.05)$

a, b, c,d means in the same row with different superscripts are significantly different $(\mathrm{P}<0.05)$ 
The same trend was showed by Sugimoto et al. (2007) who found that ruminal $\mathrm{NH}_{3}-\mathrm{N}$ was higher in Potato pulp silage with urea than that without urea. Total VFA's concentrations in the rumen liquor at $2 \mathrm{~h}$ and $4 \mathrm{~h}$ post feeding was significantly $(\mathrm{P}<0.05)$ higher than at $0 \mathrm{~h}$ time of all rations. The increase of VFA's in the rumen after feeding due to the fermentation of feed carbohydrates to VFA's. Moreover, the silage is containing VFA's. Also, lactic acid from silage is converting to propionic acid in the rumen as mentioned by Kung et al. (2018). Sugimoto et al. (2008) noticed that total VFA concentrations was increased after feeding and ranged from 9 to $11 \mathrm{mmol} / \mathrm{dL}$ at $2 \mathrm{~h}$ post feeding. The differences of total VFA among rations A, $\mathrm{B}, \mathrm{C}$ and $\mathrm{D}$ were not significant and the differences among rations $\mathrm{E}, \mathrm{F}$ and $\mathrm{G}$ were not significant. Total VFA's of rations containing silages with urea was significantly $(\mathrm{P}<0.05)$ higher than control and rations containing silages without urea. The same trend was noticed by Sugimoto et al. (2007). The differences of Microbial protein among all rations were not significant except ration $\mathrm{B}$ was lower than other rations. However, the synthesis of rumen microbial protein can be affected by synchronizing of energy releasing by fermentation of carbohydrates and $\mathrm{N}$ availabilities from nitrogen sources in the rumen as reported by Harun and Sali (2019) and Pathak (2008).

\section{CONCLUSION}

It could be concluded that:

1. Non-commercial tubers and roots such as small and very large, broken and unmarketable of Potato, sweet potato and turnip could be ensiling with crop residues such as rice straw and wheat straw.

2. The suitable silages should be containing about $25 \%$ dry crop residues such as rice straw and $75 \%$ fresh tubers or roots.

3. The feeding $60 \%$ of requirements from concentrate feed mixture (CFM) with silages of potato, sweet potato and turnip mixing with rice straw was suitable and safe rations for feeding sheep.

4. The use of urea as a silage additive is requiring more studies.

5. Further studies are recommended to evaluate silages of tuber and roots and its by- products on animal performance and its economical return.

\section{REFERENCES}

Abo-Donia, F. M. A., U. A. Al-Zalaki, A. Aiad and G. H. Zaza (2004). Effect of using different sources of dietary energy for making berseem silage on performance of lactating cows. J. Agric. Sci. Mansoura Univ., 29(1): 11-26.

Aibibula, Y., A. Okine, M. Hanada, S. Murata, M. Okamoto and M. Goto (2007). Effect of replacing rolled corn with potato pulp silage in grass silage-based diets on nitrogen utilization by steers. Asian-Aust. J. Anim. Sci., 20(8): 1215-1221.

Alberta Agriculture Food and Rural Development (AARD) (2004). Silage manual. C. Kaulbars and C. King, eds., Information Packaging
Centre, Edmonton, AB.

Ali, A. H., M. R. Shafeek, R. A. Mahmoud and M. ElDesuki (2014). Effect of various levels of organic fertilizer and humic acid on the growth and roots quality of turnip plants (Brassica rapa). Current Science International, 3(1): 714.

Altinok, S. and A. Karakaya (2003). Effect of Growth Season on Forage Yields of Different Brassica Cultivars Under Ankara Conditions. Turk J. Agric., 27: 85-90.

AOAC, Association of Official Analytical Chemists (1995). Official Methods of Analysis, $16^{\text {th }}$ ED. Washington, D.C, USA.

AOAC, Association of Official Analytical Chemists (2016). Official Methods of Analysis, 20 ${ }^{\text {th }}$ ED. Washington, D.C, USA.

Babaeinasab, Y., Y. Rouzbehan, H. Fazaeli and J. Rezaei (2015). Chemical composition, silage fermentation characteristics, and in vitro ruminal fermentation parameters of potatowheat straw silage treated with molasses and lactic acid bacteria and corn silage. J. Anim. Sci., 93(9): 4377-4386.

Charmley, E., D. Nelson and F. Zvomuya (2006). Nutrient cycling in the vegetable processing industry: utilization of potato by-products. Can. J. Soil Sci., 86: 621-629.

Conway, E. J. (1957). Microdiffusion analysis and Volumetric Error Rev. Ed. Lockwood, London.

Duncan, D.B. (1955). Multiple range and multiple Ftest. Biometrics', 11: 1-42.

Giang, H. H., L. V. Ly and B. Ogle (2004). Evaluation of ensiling methods to preserve sweet potato roots and vines as pig feed. Livestock Research for Rural Development Vol. 16(7): paper 45.

Hadgu, G. Z., T. Negesse and A. Nurfeta (2015). Nutritive value of fresh, dried (hay) and ensiled vines of four sweet potato (Ipomoea batatas) varieties grown in southern Ethiopia. Tropical and Subtropical Agroecosystems, 18(2): 195205.

HaiYan, C., Y. Bin, S. HongXia, Z. LiQingm, W. XiaoQing, S. ZhanQuan and L. JianXin (1998). Fermentation characteristics and rumen degradation of turnip ensiled with rice straw. Acta Agriculturae Zhejiangensis, 10(4): 215 219 (abstract).

Hart, S. P. and F. P. Horn (1987). Ensiling characteristics and digestibility of combinations of turnips and wheat straw. J. Anim. Sci., 64(6): 1790-1800.

Harun, A. Y and K. Sali (2019). Factors affecting rumen microbial protein synthesis: a review. Vet. Med. Open J., 4(1): 27-35.

Hough, R. L., M. H. Wiedenhoeft, B. A. Barton and A. C. Thompson Jr. (1994). The effect of dry matter level on effluent loss and quality parameters of potato-based silage. J. Sustainable Agric. 4(2):53-63.

Jacobs, J. L., G. N. Ward, A. M. Mcdowell and G. A. Kearney (2001). A survey on the effect of establishment techniques, crop management, 
moisture availability and soil type on turnip dry matter yields and nutritive characteristics in Western Victoria. Australian Journal of Experimental Agriculture, 41,743-751.

Kleinschmit, D. H. and L. Kung Jr. (2006). A Metaanalysis of the effects of Lactobacillus buchneri on the fermentation and aerobic stability of corn and grass and small-grain silages. J. Dairy Sci., 89 (10): 4005-4013.

Kung, Jr. L., R. D. Shaver, R. J. Grant and R. J. Schmidt (2018). Silage review: Interpretation of chemical, microbial, and organoleptic components of silages. J. Dairy Sci., 101(5): 4020-4033.

Leonel, M., E. L. Do Carmo, A. M. Fernandes, R. P. Soratto, J. A. M. Ebúrneo, É. L. Garcia and T. P. R. dos Santos (2017). Chemical composition of potato tubers: the effect of cultivars and growth conditions. Food Sci. Technol., 54 (8): 2372-2378.

Mahanna, B. (1994). Proper management assures highquality silage, grains. Feedstuffs, n.10/17, p.12-56 (cited from Rigueira et al., 2013).

McDonald, P., R. A. Edwards, J. F. D. Greenhalgh, C. A. Morgan, L.A. Sinclair and R. G. Wilkinson (2010). Animal Nutrition. $7^{\text {th }}$ ed. London, UK, pp. 505-506.

Mutavhatsindi T. F., B. D. Nkosi, J. J. Baloyi and T. Langa (2018). Effects of a fibrolytic enzyme and bacterial inoculants on the fermentation, chemical composition and aerobic stability of ensiled potato hash. South. Afr. J. Anim. Sci., 48(2): 244-252.

Nelson, M. L., J. R. Busboom, J. D. Cronrath, L. Falen, and A. Blankenbaker (2000). Effects of graded levels of potato by-products in barley- and corn-based beef feedlot diets: I. Feedlot performance, carcass traits, meat composition, and appearance. J. Anim. Sci., 78(7): 18291836.

Nicholson, J. W. G. and L. B. Macleod (1966). Effect of form of nitrogen fertilizer, A preservative, and a supplement on the value of high moisture grass silage. Can, J. Animal Sci, 46(2): 71-82.

Nkosi, B. D. and R. Meeske (2010). Effects of whey and molasses as silage additives on potato hash silage quality and growth performance of lambs. South African J. Anim. Sci., 40(3): 229237.

Nkosi, B. D., R. Meeske and I. B. Groenewald (2010). Effects of ensiling potato hash with either whey or sugar cane molasses on silage quality and nutrient digestibility in sheep. Livestock Research for Rural Development, 22(1): 1-9.

NRC (1985). Nutrient Requirements of sheep. $6^{\text {th }}$ ED; National Academy of Science, National Research Council, Washington, DC.

Okine A., M. Hanada, Y. Aibibula and M. Okamoto (2005). Ensiling of potato pulp with or without bacterial inoculants and its effect on fermentation quality, nutrient composition and nutritive value. Anim. Feed Sci. and Tech., 121(3-4): 329-343.
Osman, A. A., E. S. Soliman, F. Z. Swidan and A. N. Ismail (2007). Evaluation of different ratios of citrus pulp and some crop residues silages. Egyptian J. Nutrition and feeds, 10(2) Special issue: $231-243$.

Pathak A. K. (2008). Various factors affecting microbial protein synthesis in the rumen. Veterinary World, Vol. 1(6): 186-189.

Pen, B. T. Iwama, M. Ooi, T. Saitoh, K. Kida, T. Iketaki, J. Takahashi and H. Hidari (2006). Effect of potato by-products-based silage on rumen fermentation, methane production and nitrogen utilization in Holstein steers. AsianAust. J. Anim. Sci., 19(9): 1283-1290.

Penno, J. W., A. M. Bryant, A. R. Napper and P. J. A. Copeman (1996). The effect of feeding turnips to dairy cows grazing limited amounts of pasture in mid to late lactation on milk solids production Proceedings of the New Zealand Society of Animal Production, Vol. 56: 242244.

Research Institute for cattle feeding at Hoorn, Holland (1961). Some determinations for judging the silage quality (C.F. Nowar.1969. Nowar, M.Sc. Thesis, Fac. Agric., Cairo Univ.).

Rigueira, J. P. S., O. G. Pereira, K. G. Ribeiro, H. C. Mantovani and M. C. N. Agarussi (2013). The chemical composition, fermentation profile, and microbial populations in tropical grass silages. Revista Bras. Zootec., 42(9): 612-621.

Rui-rui S., L. Qiu-feng, L. Yun-qi, S. Xiao-yu, C. Yufeng, Y. Kang-ning, W. Yong-sheng, W. Meimei, G. Yan-xia and L. Jian-guo (2018). Effect of additives on fermentation quality and cattle rumen degradability of mixed potato pulp and corn straw silage. Acta Prataculturae Sinica, 27(11): 200-208.

Ruiz, M. E, E. Lozano and A. Ruiz (1981). Utilization of sweet potatoes (Ipomoea batata (1.) Lam) in animal feeding III. The addition of various levels of roots and urea to sweet potato forage silages1, Trop Anim Prod, 6(3): 234-244.

Sadri, K., Y. Rouzbehan, H. Fazaeli and J. Rezaei (2018). Influence of dietary feeding different levels of mixed potato-wheat straw silage on the diet digestibility and the performance of growing lambs. Small Ruminant Research, 159: 84-89.

Samy, M. M., M. G. Abd El-Aziz and N. A. Mohamed (2014). Effect of some agricultural treatments on some sweet potato cultivars. Middle East J. Agric. Research, 3(4): 1221-1231.

Shultz, T. A and E. Shultz (1970). Estimation of rumen microbial nitrogen by three analytical methods. J. Dairy Sci., 53: 781-784.

SPSS Statistics V24.0. IBM Corporate headquarters1 New Orchard Road Armonk, New York 10504-1722 United States US: 914-499-1900.

Sugimoto, M., S. Waka and M. Ooi (2010). The effects of urea-treated potato pulp (pp) ensiled with beet pulp or wheat bran pellets to reduce moisture of $p p$ and flake density of corn grain supplemented with the pp silage on 
digestibility and ruminal fermentation in beef steers. Anim. Sci. J., 81(3): 316-324.

Sugimoto, M., M. Kanamoto, T. Chiba, H. Hidari, K. Kida, W. Saito, M. Ooi, Y. Sato and T. Saito (2008). The effects of protein sources supplemented with urea-treated potato pulp (PP) silage and feeding levels of the PP silagebased concentrate on feed intake, digestibility and ruminal fermentation in beef steers. Animal Sci. J., 79(4): 443-452.

Sugimoto, M., T. Chiba, M. Kanamoto, H. Hidari, K. Kida, W. Saito, M. Ooi, Y. Sato and T. Saito (2007). Effects of urea treatment of potato pulp and inclusion levels of Potato Pulp silage in supplements on digestibility and ruminal fermentation in beef steers. Anim. Sci. J., 78(6): 587-595.

Waldo, D. R. and L. H. Schultz (1956). Lactic acid production in the rumen. J. Dairy Sci., 39: 1455.

Warner, A. C. J. (1964). Production of volatile fatty acids in the rumen. Methods of measurements Nutr. Abstr. \& Rev. B, 34: 339.

Zobell, D. R, E. K. Okine, K. C. Olson, R. D. Wiedmeier, L. A. Goonewardene and C. Stonecipher (2005). Effects of feeding wheat straw and middlings ensiled with whey on digestibility and growth of cattle. Can. J. Anim. Sci., 85: 69-74.

\section{تقييم سيلاج البطاطس والبطاطاواللقت مع قش الأرز أو تبن القمح مع أو بدون اليوريا ودراسة تأثير العلائق المحتوية على السيلاج على معاملات الهضم وتخمرات الكين الكرش في الأغنام \\ محمد عبد العليم أحمد زكى، أحمد أحمد عثمان 1، السيا سليمان محمد سليمان2، عبد الغنى حسانين عبد الغتى2 2

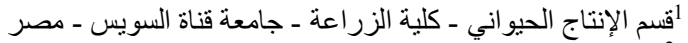

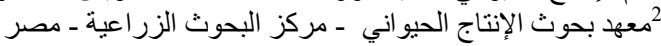

أجريت هذه الدراسة بهدف تقييم سيلاج درنات البطاطس وجذور البطاطا وجذور اللفت الغير تجارية. نم عمل السيلاج في التجربة

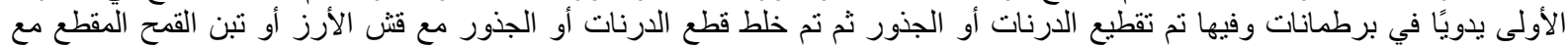

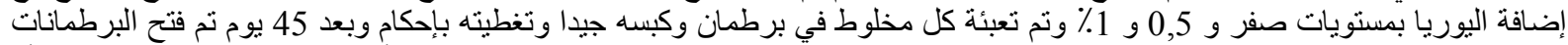

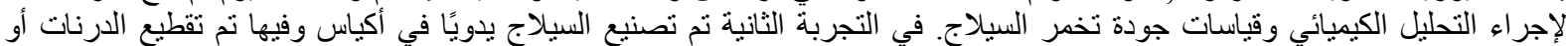

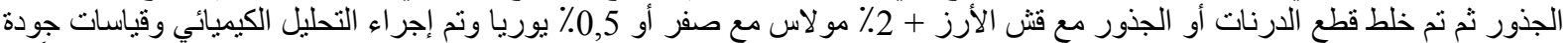

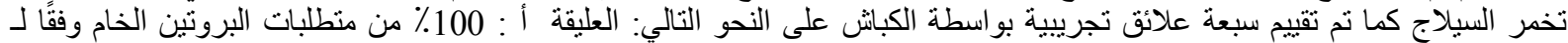

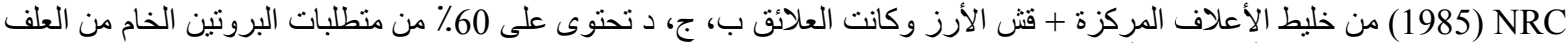

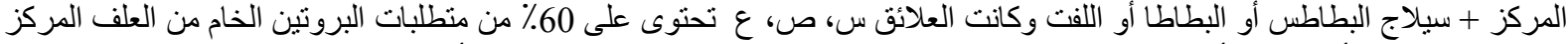

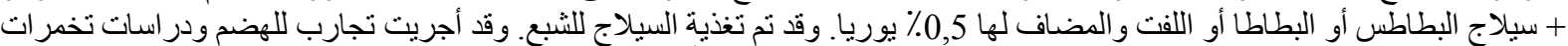

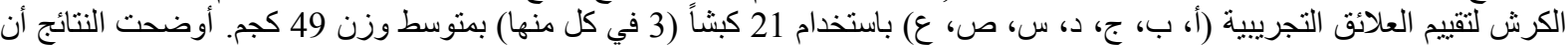

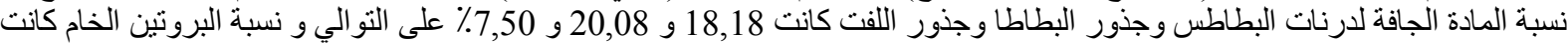

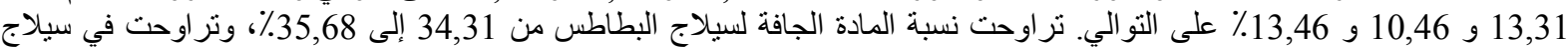

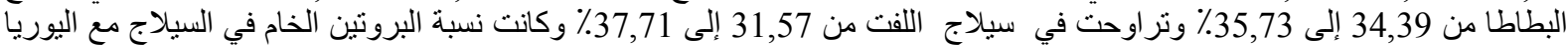

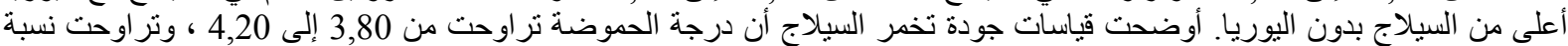

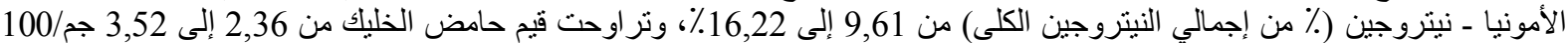

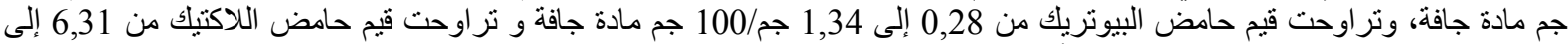

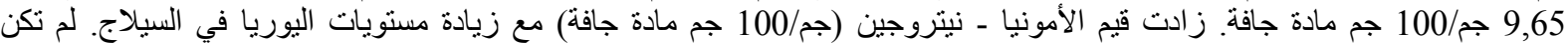

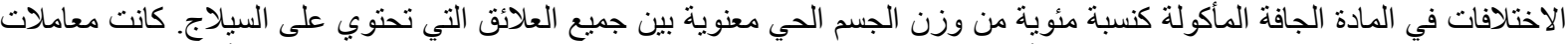

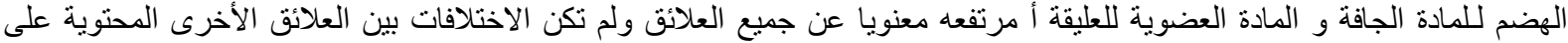

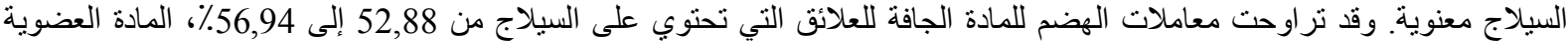

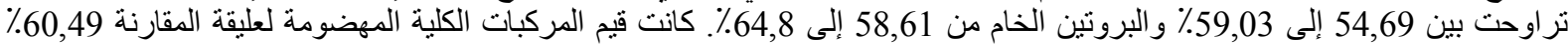

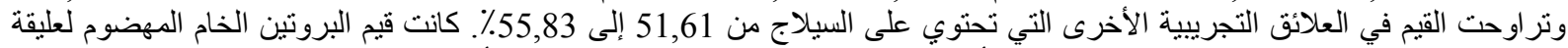

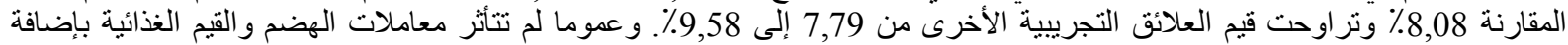

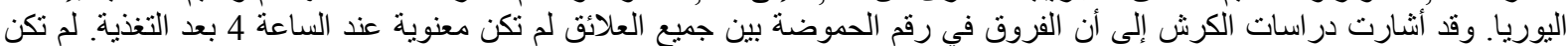

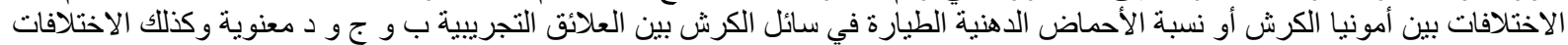

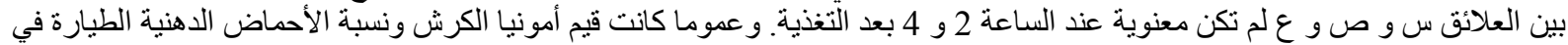

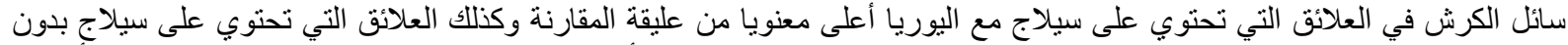

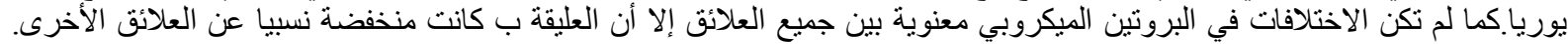

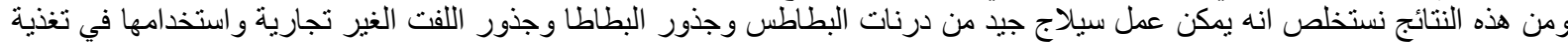

